\title{
Extraction and Distortion Rectification of Signboards in a Scene Image for Robot Navigation
}

\author{
Non-member Yongmei Liu \\ (BMC, RIKEN) \\ Non-member Tsuyoshi Yamamura (Aichi Prefectural University) \\ Non-member Toshimitsu Tanaka (Nagoya University) \\ Member Noboru Ohnishi (Nagoya University \& BMC, RIKEN)
}

\begin{abstract}
In this paper, we describe an approach for finding signboard information in a grey scene image and rectifying a distorted signboard image taken from a nonorthogonal viewing direction. First, signboards in scene images are detected using several heuristics of characters and character lines. Then, we attempt to compute the orientation of the camera relative to a detected signboard from a single view. Finally, we rectify the distortion of signboard images, making the recognition of characters under a wider range of viewing conditions much easier. We show experimental results that demonstrate the effectiveness of the proposed approach. Lastly, we present an application of the proposed method to robot navigation using signboards on walls and doors as landmarks, where we could navigate a mobile robot in indoor environments successfully. Using signboards in scenes as landmarks enables us to realize humanlike navigation and map generation, and also benefits human-robot communication.
\end{abstract}

Keywords: Character Extraction, Camera Orientation Estimation, Distortion Rectification and Mobile Robot Navigation

\section{Introduction}

Character information plays an important role in our everyday life. There is much character information such as signboards and traffic (road) signs around us. In real life, we humans can separate these characters from other objects, get necessary information from them immediately, and use the information to reach our destination. If signboards in scene images could also be recognized, the information could be applied to vision-based mobile robot systems.

In this study, we propose an approach of extraction and distortion rectification of signboards in a grey scene image for mobile robot navigation. In recent years, there has been some approaches on extracting characters from scene images: minimization of a cost function, which reflects some features specific to Japanese printed characters ${ }^{(1)}$; use of color segmentation and spatial variance to locate text in $C D$ and book cover images $^{(2)}$; and extraction of character pattern candidates using position and grey-level information ${ }^{(3)}$. In the real world, characters in scene images originally exist in 3-D space; thus, they can be distorted by changing the camera viewing direction. These systems, however, do not deal with 3-D distortion due to the slanting of the viewing direction. Here, we describe an approach for finding character information in a scene image along with the camera viewing direction relative to the found characters, and rectifying a distorted signboard image in order to make it easily recognizable.

In this paper, we concentrate on the approach for es- timating the camera viewing direction and rectifying a distorted signboard image. Section 2 is a survey of character-based mobile robot navigation system. Section 3 gives a brief description of the algorithm for detecting character information from a scene image with experimental results. Section 4 presents two methods to compute the relative orientation between the camera and a detected signboard as well as methods of rectifying a distorted signboard image. Section 5 shows the experimental results with discussion. Section 6 is an example of the application to an indoor mobile robot navigation system and the conclusion is given in Section 7 .

\section{Character-Based Robot Navigation}

In recent years, there has been increasing interest in the use of robots to perform ordinary tasks that require mobility in environments such as office buildings, hospitals and factories. In these works, natural landmarks, such as walls, doors and corners, or artificial landmarks, such as stripes on the floor and man-made signs of special shapes or colors, are used as external reference sources for the robot. However, the shape, size and location of these natural landmarks often depend on the environment and are not unique, making these systems lack certainty and flexibility ${ }^{(4)-(6)}$. On the other hand, artificial landmarks often require improvement of the environment, resulting in a system with very little flexibility ${ }^{(7)(8)}$. In real life, landmarks are often marked in maps in the form of characters, and we often identify these landmarks in environments by 
means of signboard recognition. Thus, character information can also be considered as a valuable and reliable clue in robot navigation systems. In our research, we are aiming at building a character-based mobile robot navigation system.

The mobile robot, shown in Fig.1, is a B12 mobile robot platform manufactured by Real World Interface Inc. It is equipped with 12 sonar sensors, and a forwardlooking Sony CCD camera (Sony XC-003) is mounted on top of the robot. Both sonar information and camera images are processed by an external computer (SGI ONYX). The robot communicates with the external computer via RS-232c cables.

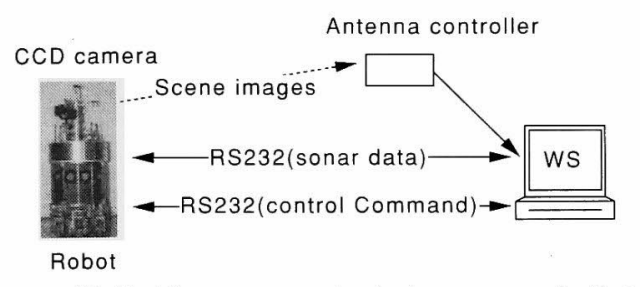

Fig. 1. Robot system architecture

When the robot explores its environment for the first time, it will generate an environment map. While moving in its workspace, from the data of sonar sensors, the robot can detect walls, corners and junctions, and with these information the topology of a hallway can be obtained. At each junction, if the robot recognizes any signboards, it will record the signboard in the environment map. Finally, the robot builds an environment map that integrates topological information of the environment, approximate distance information, plus signboard information. Once a character-based environment map is formed, the robot is able to use this information in order to navigate reliably from place to place. Using signboards, a robot can identify the objects of interest (such as meeting room and emergency exit) in its environment. Therefore, the task to be accomplished by the robot can be specified in high-level semantic terms, such as "go down the hallway, turn right at the meeting room and stop at the emergency exit", and human-robot communication will be facilitated. This will also enable us to realize human-like navigation and map-generation.

\section{Finding Character Information in a Scene Image}

Generally, Japanese characters (Japanese Kana and Chinese characters) displayed on signboards and on traffic (road) signs have the following features: high spatial frequency, high grey-level contrast, constant aspect ratio, and geometrical features of character arrangement. We use these features to extract the character components from scene images. First, we extract subregions with high spatial frequency and large variance in grey levels as candidates of character components from an input scene image. Then, we select characters by using several heuristics such as constraints of size and shape, bimodality of an intensity histogram, alignment and proximity of characters. Details of the character detection process are described in Ref. ${ }^{(9)}$.
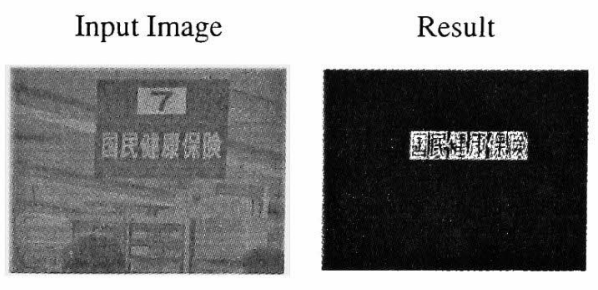

Indoor No.10

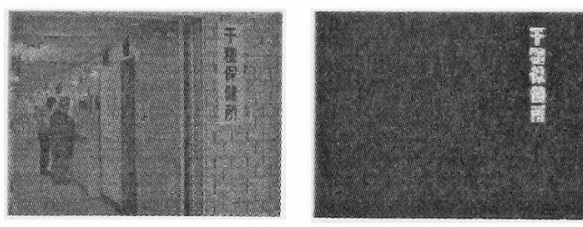

Indoor No.20

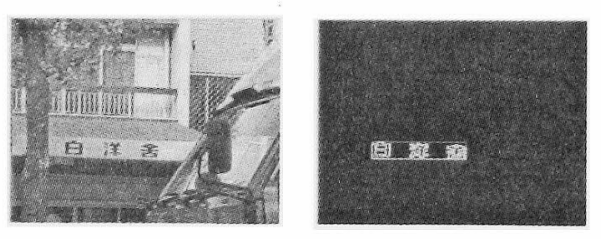

Outdoor No.14
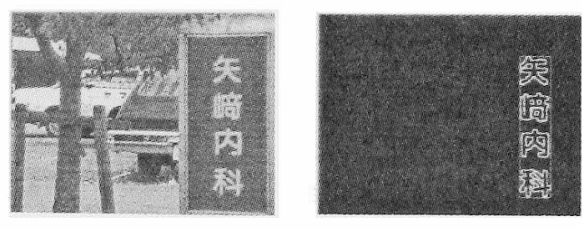

Outdoor No.16

Fig. 2. Experimental Results of Character Detection (60 images)

We conducted experiments on 40 outdoor and 20 indoor scene images. The experimental results are shown in Table 1. Figure 2 shows some examples of experimental results on real scene images.

Table 1. Experimental Results

\begin{tabular}{|c|c|c|c|c|}
\hline & Number & $\begin{array}{c}\text { Number of } \\
\text { Extraction }\end{array}$ & $\begin{array}{c}\text { Rate of } \\
\text { Extraction }\end{array}$ & Err. \\
\hline Char. & 397 & 351 & $88.4 \%$ & 23 \\
Char. line & 77 & 63 & $81.8 \%$ & 12 \\
\hline
\end{tabular}

\section{Orientation Estimation and Distortion Rec- tification}

Once a signboard is extracted from a scene image, the next important task is to determine the relative orientation of the camera and rectify a distorted signboard. Because estimating the 3 -D structure of a scene from a single visual image is impossible without certain assumptions regarding the scene and the projection geometry, in this paper we use only one restriction that 


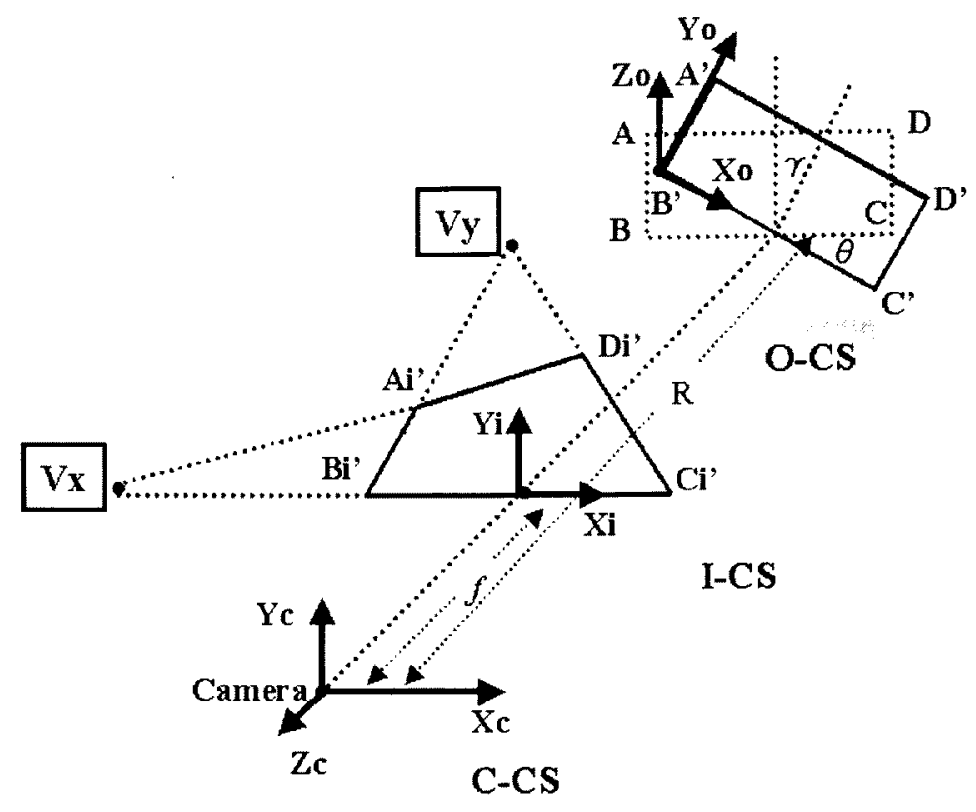

Fig. 3. Coordinate Systems

the signboard is rectangular. This is not very restrictive for real applications, because many signboards in indoor or outdoor environment satisfy this constraint. Once a character line is detected, we seek objects that surround these characters and if the edge of an object satisfied some restrictions (such as made up straight lines, proper relative size and location to the detected character line), we select it as the signboard (rectangle).

It is well known that under perspective imaging vanishing points of parallel lines can be used as useful cues to infer information about a $3-D$ scene from a single view, including estimating the orientation of planar surfaces and obtaining a 3-D interpretation of a scene. This method has been widely used in previous researches. In ${ }^{(10)}$, vanishing points are used to estimate surface orientation, and in ${ }^{(11)},{ }^{(12)}$ and ${ }^{(13)}$, the positions of vanishing points are employed to understand 3-D shapes and to reconstruct or to generate 3-D models. However, these researches do not deal with the problem of distortion rectification of their 2-D representations. In our work, we use vanishing points of two pair parallel signboard edges in a scene image to estimate the camera viewing direction. Using the obtained camera viewing direction, we extends the vanishing points method to rectify a distorted signboard image by the back-projection from the image plane to the world plane. In our method, only the focal length of the camera is needed.

We also proposed another method to rectify a distorted signboard by calculating the projection transformation from the world plane to image plane without any other additional information. Unlike method in (14) (15) no Euclidean world coordinates of any image points are required, and neither camera parameters nor other external information is needed.

4.1 Coordinate systems Taking the optical center of the camera (with a focal length of $f$ ) as the origin, the coordinate systems, as shown in Fig.3, are C-CS (the camera coordinate system) and I-CS (the image coordinate system). The rectangle $A B C D$ represents a signboard which is orthogonal to the viewing direction of the camera, the rectangle $A^{\prime} B^{\prime} C^{\prime} D^{\prime}$ is the position of $A B C D$ undergoing two rotations (one around the $\mathrm{X}$ axis by an angle of $\gamma$, and the other around the $\mathrm{Y}$ axis by an angle of $\theta$ ), and the rectangle $A_{i}^{\prime} B_{i}^{\prime} C_{i}^{\prime} D_{i}^{\prime}$ is the projection of $A^{\prime} B^{\prime} C^{\prime} D^{\prime}$ on the image plane. Here, we introduce another coordinate system, O-CS (the object coordinate system, the $X_{o}-Y_{o}$ plane lies on the $A^{\prime} B^{\prime} C^{\prime} D^{\prime}$ plane, and $Z_{o}$ is the normal).

4.2 Vanishing point method The convergence of three-dimensional parallel lines under perspective projection can be used to infer information about a three-dimensional scene. Here, we use the vanishing points of the edges of a signboard to estimate the orientation of a detected signboard plane relative to the camera viewing direction from a single image.

In Fig.3, let ( $\mathrm{a}, \mathrm{b},-\mathrm{R})$ be the coordinates of point $A$ in the C-CS. Then, the coordinates of $A^{\prime}$ can be presented as

$$
\left\{\begin{array}{l}
X_{A^{\prime}}=a \cos \theta+b \sin \gamma \cos \theta \\
Y_{A^{\prime}}=b \cos \gamma \\
Z_{A^{\prime}}=-R+a \sin \theta-b \sin \gamma \cos \theta
\end{array}\right.
$$

Based on perspective transformation, the coordinates of $A^{\prime}$ in the I-CS $\left(A_{i}^{\prime}\right)$ can also be calculated using 
Eq.(1).

$$
\left\{\begin{array}{l}
x_{A^{\prime}}=\frac{f(a \cos \theta+b \sin \gamma \cos \theta)}{R-(a \sin \theta-b \sin \gamma \cos \theta)} \\
y_{\mathcal{A}^{\prime}}=\frac{f(b \cos \gamma)}{R-(a \sin \theta-b \sin \gamma \cos \theta)}
\end{array}\right.
$$

where $f$ denotes the focal length of the camera. Using Eqs.(2) and (3), we can obtain the coordinates of vanishing points $v_{x}$ and $v_{y}$.

$$
\begin{aligned}
& v_{x}(x, y)=\lim _{a \longrightarrow \infty}\left(\frac{f(a \cos \theta+b \sin \gamma \cos \theta)}{R-(a \sin \theta-b \sin \gamma \cos \theta)},\right. \\
& \left.\frac{f(b \cos \gamma)}{R-(a \sin \theta-b \sin \gamma \cos \theta)}\right)=\left(\frac{-f}{\tan \theta}, 0\right) \\
& v_{y}(x, y)=\lim _{b \longrightarrow \infty}\left(\frac{f(a \cos \theta+b \sin \gamma \cos \theta)}{R-(a \sin \theta-b \sin \gamma \cos \theta)},\right. \\
& \left.\frac{f(b \cos \gamma)}{R-(a \sin \theta-b \sin \gamma \cos \theta)}\right)=\left(-f, \frac{f}{\tan \gamma \cos \theta}\right)
\end{aligned}
$$

The location of vanishing points $v_{x}$ and $v_{y}$ can also be extracted from the image by finding the intersection points of $D_{i}^{\prime} A_{i}^{\prime}, C_{i}^{\prime} B_{i}^{\prime}$ and $B_{i}^{\prime} A_{i}^{\prime}, C_{i}^{\prime} D_{i}^{\prime}$ in I-CS.

If the focal length of the camera $(f)$ is known, the orientation of the camera viewing direction relative to a detected signboard (the values of $\gamma$ and $\theta$ ) can be computed using Eqs.(2) and (3).

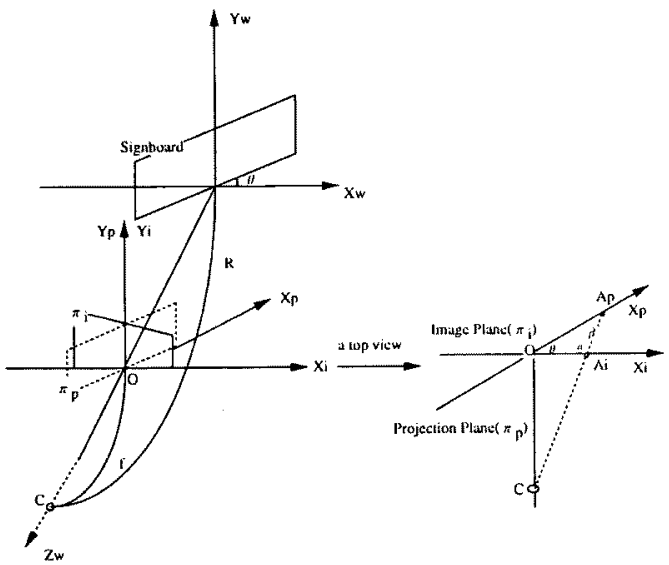

Fig. 4. Geometric Relationship in Back-Projection

Using the obtained values of $\gamma$ and $\theta$, we rectify the distorted signboard image by back-projecting it onto a plane which is parallel to the signboard plane in the 3 $\mathrm{D}$ environment. The projective structure is computable from the correspondences of image plane and the projection plane. Figure 4 shows the geometric relationship in this back-projection transformation. In Fig.4, $\pi_{i}$ and $\pi_{p}$ represent the image plane and the projection plane, respectively. Consider $A_{i}\left(x_{i}, y_{i}\right)$ to be a point in plane $\pi_{i}$, and $A_{p}\left(x_{p}, y_{p}\right)$ is its projected point in plane $\pi_{p}$. Then, the coordinate value of $A_{p}$ in plane $\pi_{P}$ can be calculated in triangular $\mathrm{O} A_{i} A_{p}$ using

$$
x_{p}=\frac{x_{i} \cdot \sin \alpha}{\sin \beta}
$$

where

$$
\alpha=\arctan \frac{O A_{i}}{O C}+90^{\circ} \text {, }
$$

$\mathrm{OC}=f$ (focal length of camera), $O A_{i}=x_{i}$ (coordinate value in the image plane), $\theta$ is the orientation of the signboard relative to the camera which is obtained by the method described above, and $\beta=180^{\circ}-\alpha-\theta$.

According to the perspective projection, we can also calculate $y_{p}$ using

$$
y_{p}=\frac{y_{i} \cdot x_{p} \cdot \cos \theta}{x_{i}}
$$

Using Eqs.(4) and (5), a distorted signboard image can be reconstructed.

4.3 Vertex coordinates method The orientation of the camera relative to the signboard can also be obtained by computing the normal vector of the signboard. Here, we attempt to obtain the normal vector orthogonal to a signboard surface from the camera coordinates of its four vertices directly. On the assumption that the shape of the signboard is rectangular, in the camera coordinate system (C-CS), as shown in Fig.3,

$$
\overrightarrow{A^{\prime} D^{\prime}}=\overrightarrow{B^{\prime} C^{\prime}}
$$

Based on coordinate relations between points in I-CS $\left(x_{i}, y_{i}\right)$ and points in C-CS $\left(X_{i}, Y_{i}, Z_{i}\right)$, Eq. (6) can be expressed as

$$
\left\{\begin{array}{l}
\frac{x_{A^{\prime}} \cdot Z_{A^{\prime}}}{f}-\frac{x_{D^{\prime}} \cdot Z_{D^{\prime}}}{f}=\frac{x_{B^{\prime}} \cdot Z_{B^{\prime}}}{f}-\frac{x_{C^{\prime}} \cdot Z_{C^{\prime}}}{f} \\
\frac{y_{A^{\prime}} \cdot Z_{A^{\prime}}}{f}-\frac{y_{D^{\prime}} \cdot Z_{D^{\prime}}}{f}=\frac{y_{B^{\prime}} \cdot Z_{B^{\prime}}}{f}-\frac{y_{C^{\prime}} \cdot Z_{C^{\prime}}}{f} \\
Z_{A^{\prime}}-Z_{D^{\prime}}=Z_{B^{\prime}}-Z_{C^{\prime}}
\end{array}\right.
$$

letting $Z_{D^{\prime}}=\mathrm{H}$, the value of $Z_{A^{\prime}}$ can be calculated from Eq.(8) as

$$
\begin{aligned}
& Z_{A^{\prime}}=H . \\
& \frac{\left(y_{C^{\prime}}-y_{D^{\prime}}\right) x_{B^{\prime}}+\left(y_{D^{\prime}}-y_{B^{\prime}}\right) x_{C^{\prime}}+\left(y_{B^{\prime}}-y_{C^{\prime}}\right) x_{D^{\prime}}}{\left(x_{C^{\prime}}-x_{B^{\prime}}\right) y_{A^{\prime}}+\left(x_{A^{\prime}}-x_{C^{\prime}}\right) y_{B^{\prime}}+\left(x_{B^{\prime}}-x_{A^{\prime}}\right) y_{C^{\prime}}}
\end{aligned}
$$

The values of $Z_{B^{\prime}}, Z_{C^{\prime}}$ can also be determined similarly as

$$
\begin{aligned}
& Z_{B^{\prime}}=H \\
& \frac{\left(y_{C^{\prime}}-y_{D^{\prime}}\right) x_{A^{\prime}}+\left(y_{D^{\prime}}-y_{A^{\prime}}\right) x_{C^{\prime}}+\left(y_{A^{\prime}}-y_{C^{\prime}}\right) x_{D^{\prime}}}{\left(x_{C^{\prime}}-x_{B^{\prime}}\right) y_{A^{\prime}}+\left(x_{A^{\prime}}-x_{C^{\prime}}\right) y_{B^{\prime}}+\left(x_{B^{\prime}}-x_{A^{\prime}}\right) y_{C^{\prime}}}
\end{aligned}
$$

$$
\begin{aligned}
& Z_{C^{\prime}}=H \\
& \frac{\left(y_{B^{\prime}}-y_{D^{\prime}}\right) x_{A^{\prime}}+\left(y_{D^{\prime}}-y_{A^{\prime}}\right) x_{B^{\prime}}+\left(y_{A^{\prime}}-y_{B^{\prime}}\right) x_{D^{\prime}}}{\left(x_{C^{\prime}}-x_{B^{\prime}}\right) y_{A^{\prime}}+\left(x_{A^{\prime}}-x_{C^{\prime}}\right) y_{B^{\prime}}+\left(x_{B^{\prime}}-x_{A^{\prime}}\right) y_{C^{\prime}}}
\end{aligned}
$$


Then, we can obtain the $\mathrm{X}$ and $\mathrm{Y}$ coordinates of the four vertices of the signboard in the C-CS (with a coefficient of $\mathrm{H}$ ). Thus, the relative coordinates of points $A^{\prime}, B^{\prime}, C^{\prime}$ and $D^{\prime}$ in the C-CS are acquired. The unit normal vector of the signboard plane can be given as

$$
\vec{n}=\frac{\overrightarrow{A^{\prime} B^{\prime}} \times \overrightarrow{B^{\prime} C^{\prime}}}{\left|\overrightarrow{A^{\prime} C^{\prime}} \times \overrightarrow{B^{\prime} C^{\prime}}\right|}
$$

This gives an alternative method for estimating the orientation of a signboard plane.

Based on the results obtained above, we developed another approach to rectify a distorted signboard. First we attempt to obtain the transformation matrix between the camera coordinate system (C-CS) and the object coordinate system (O-CS). The 3-D transformation can be represented as

$$
\left[\begin{array}{c}
X_{c} \\
Y_{c} \\
Z_{c} \\
1
\end{array}\right]=\left[\begin{array}{cccc}
a 11 & a 12 & * & b 1 \\
a 21 & a 22 & * & b 2 \\
a 31 & a 32 & * & b 3 \\
0 & 0 & 0 & 1
\end{array}\right]\left[\begin{array}{c}
X_{o} \\
Y_{o} \\
Z_{o} \\
1
\end{array}\right]
$$

Because we are only concerned with points in the signboard plane $\left(X_{0}-Y_{o}\right.$ plane $)$, we can ignore the values in the third column of the transformation matrix. Thus, the transformation can be defined uniquely by using three noncollinear points. As the coordinates of $A^{\prime}, B^{\prime}$, $C^{\prime}$ and $D^{\prime}$ in C-CS have been obtained, their corresponding positions in O-CS can also be calculated as

$$
\left\{\begin{array}{l}
A^{\prime}\left(0,\left|\overrightarrow{A^{\prime} B^{\prime}}\right|, 0\right) \\
B^{\prime}(0,0,0) \\
C^{\prime}\left(\left|\overrightarrow{B^{\prime} C^{\prime}}\right|, 0,0\right) \\
D^{\prime}\left(\left|\overrightarrow{B^{\prime} C^{\prime}}\right|,\left|\overrightarrow{A^{\prime} B^{\prime}}\right|, 0\right)
\end{array}\right.
$$

Substituting these into Eq.(12), the transformation matrix can be determined.

Once the transformation matrix is obtained, the point correspondence between the camera coordinate system and the object coordinate system is established, and the signboard image can be rectified by calculating each coordinate in the object coordinate system.

\section{Experiments and Discussion}

5.1 Experimental results We carried out simulation experiments by calculating the angle between the camera direction and a signboard normal. By image transformation, we created images to be observed at viewing directions of $\theta=10^{\circ}, 20^{\circ}, 30^{\circ} \ldots$ and $\gamma=$ $10^{\circ}, 20^{\circ}, 30^{\circ} \ldots$ (the maximum value of both $\theta$ and $\gamma$ is $45^{\circ}$ ) from an original image captured from an orthogonal viewing direction $\left(\theta=0^{\circ}\right.$ and $\left.\gamma=0^{\circ}\right)$. The second column of Fig. 5 shows some examples of images generated by this method. Using the method described in 4.2 , we estimated the camera viewing direction. Table 2 shows some of the results.
As a result, the error of the estimated value of the viewing direction (degree) is less than $5^{\circ}$, and this confirms the effectiveness of the proposed method.

Using the two methods described in 4.2 and 4.3 , we also conducted experiments to rectify distorted signboard images. Figure 5 shows some examples of the results. Here, we employ a simple template matching scheme to evaluate the rectification effect. Our matching strategy is as follows. Let $T_{1}, T_{2}, \ldots, T_{k}$ be the data of binary images of each character in a signboard taken from an orthogonal viewing direction. Let $I$ be the image of a character in a signboard taken from a slanting viewing direction or a character in the rectified signboard image. Every image pair $\left(I, T_{i}\right)$ is normalized to have the same size as $T_{i}$. Here, we use the following matching coefficient between $I$ and $T_{i}$.

$$
R_{i}=\frac{\text { number of matched pixels }}{\text { number of total pixels }}
$$

Table 3 shows the average matching rates of input images, rectified images using the method in 4.2 and rectified images using the method in 4.3 . The results show that signboard rectification makes characters obtained from a nonorthogonal viewing direction much easily recognizable.

Table 2. Experimental Results on Estimating Camera Viewing Direction

\begin{tabular}{|c|c|c|c|}
\hline $\begin{array}{c}\text { Signboard } \\
\text { (number) }\end{array}$ & $\begin{array}{c}\text { 非常口 } \\
(10)\end{array}$ & $\begin{array}{c}\text { 地下鉄 } \\
(10)\end{array}$ & $\begin{array}{c}\text { 筆枒室 } \\
(10)\end{array}$ \\
\hline Average Err. & 2.13 & 1.98 & 3.76 \\
\hline
\end{tabular}

Table 3. Experimental Results on Rectifying Distortion

\begin{tabular}{|c|c|c|c|}
\hline $\begin{array}{c}\text { Signboard } \\
\text { (number) }\end{array}$ & $\begin{array}{c}\text { 非常口 } \\
(10)\end{array}$ & $\begin{array}{c}\text { 地 F 鉄 } \\
(10)\end{array}$ & $\begin{array}{c}\text { 事務空 } \\
(10)\end{array}$ \\
\hline Input Images & 0.8141 & 0.7582 & 0.6807 \\
Method of 3.2 & 0.8211 & 0.8722 & 0.7482 \\
Method of 3.3 & 0.8744 & 0.9164 & 0.7692 \\
\hline
\end{tabular}

5.2 Uncertainty Analysis Feature (here, vanishing points and vertices of a signboard) detection and extraction, whether manual or automatic (e.g. using an edge detector), can only be achieved to a finite accuracy. Here, we use some experimental examples to show how these errors propagate to the results.

Table 4. Influence of Errors in Vertices Coordinates on Rectification Results(average matching rate)

\begin{tabular}{|c|c|c|}
\hline & method in 4.2 & method in 4.3 \\
\hline before rectification & 0.7249 & 0.7249 \\
without noise & 0.7880 & 0.7650 \\
noise $(-3 \sim 3)$ & 0.7633 & 0.7482 \\
noise $(-5 \sim 5)$ & 0.7502 & 0.7405 \\
noise $(-10 \sim 10)$ & 0.6970 & 0.6940 \\
\hline
\end{tabular}

We add random noise with uniform distribution (with a scale of 3,5 and 10 pixels) to each $(x, y)$ of the four vertics, and conduct signboard rectifiction experiments using the two methods. In order to make it consistent, 


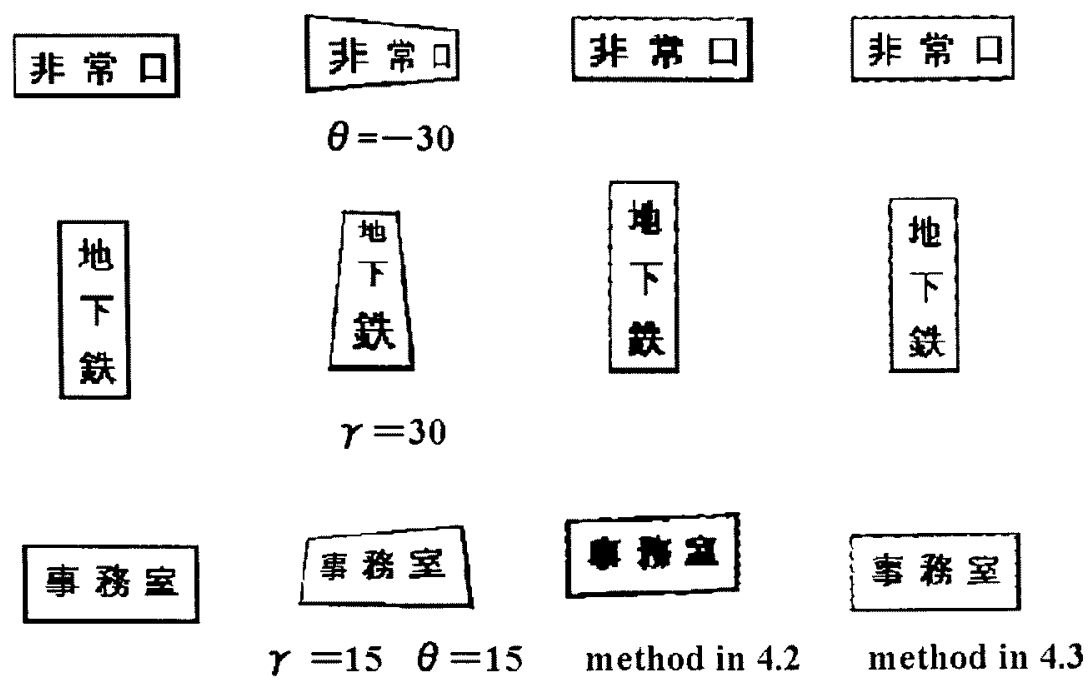

Fig. 5. Experimental Results of Distorted Signboard Rectification

in the vanishing points based method, we also only use the coordinates of four vertices to calculate the positions of vanishing points. Figure 6 shows some examples of how noise of vertices coordinates affect the rectification results. The first row of Fig. 6 are the input images, ' + ' represents the position of a vertex with an added noise. The second row shows the rectification results based on the vanishing points method, and the third row shows results based on the vertices coordinates method.

We conducted experiments on 5 images for each noise (the scale of noise is 3,5 and 10 pixels separately). Table 4 shows the average matching rates of input image, rectified images for each noise. The results show that for the input image $\left(\theta=45^{\circ}\right)$, when the average scale of noise is less than 5 pixels, the rectification method is effective(The size of the a signboard in an input image is a little different from each other, the average size is about $50 \times 130$ pixels).

5.3 Comparison of two methods In the previous sections, we proposed two methods (the vanishing point method and the vertex coordinates method) to estimate camera viewing direction and to rectify the distortion of a signboard image, and showed the experimental results obtained using the two methods. In this section, we compare the two methods.

Compared with the vertex coordinates method, the vanishing point method has a wider range of applications because its only constraint is that a signboard has two pairs of parallel edges; however, the method based on vertex coordinates requires the shape of a signboard to be a rectangular. On the other hand, the vanishing point method requires prior knowledge of the value of the camera focal length $(f)$, while the vertex coordinates method is unrelated to $f$, therefore, the size of the signboard image rectified by the vanishing point method is nearly the same as that of the original one. Conversely, the size of the signboard image rectified by the vertex coordinates method is irregular. Table 3 shows that the matching rate using the vertex coordinates method is higher than that using the vanishing point method, and this indicates that the vertex coordinates method performs better than the vanishing point method. We also find in Fig. 5 that the rectified image of “事務室” using the vanishing point method has a warp. The reason is that the input image underwent two back-projections during the rectification processing (in both $\mathrm{X}$ and $\mathrm{Y}$ direction, “非常口" only in Y direction, and "地下鉄” only in X direction), and some image information is missed. If the input image is of higher resolution, this problem can be improved to a certain extent. On the other hand, the vertex coordinates method handles all the three cases in the same way, and can avoid this weakness.

5.4 Discussion The task of getting character information from a scene image consists of three parts: first, to find character lines in a grey scene image. Here, we use several constraints of characters and character lines (such as grey-level contrast, size, shape and arrangement features). The second task is to estimate the orientation of the camera relative to a detected signboard and rectify a distorted signboard image. Once a character line is detected, we try to find the signboard boundary (rectangle), both the estimation of camera viewing direction (Eq.(2),(3) and (11)) and the distortion rectification (Eq. (4),(5) and (12)) are based on the coordinates of the boundary. One reason that we choose the signboard boundary (rectangle) as a reference is that it has two pairs of parallel edges, and this make it possible to infer the 3-D information from a single view with little information about camera cali- 


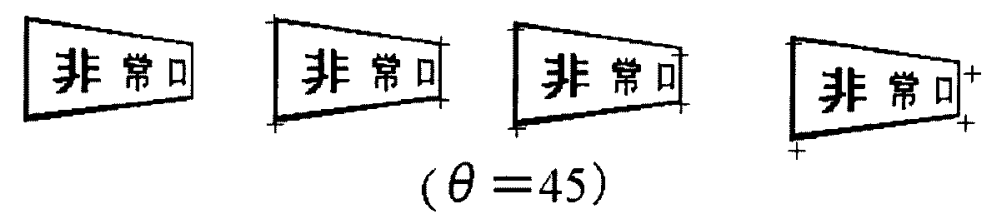

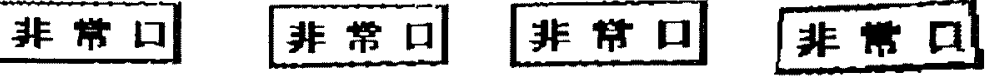 \\ Method in 4.2}

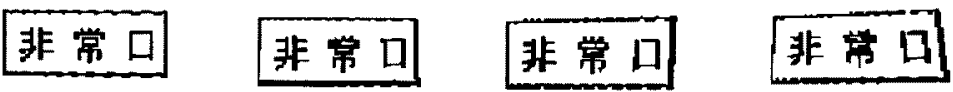

\section{Method in 4.3}
(a)noise $=0$
(b)noise $=(-3 \sim 3)$
(c) noise $=(-5 \sim 5)$
(d)noise $=(-10 \sim 10)$

Fig. 6. Rectification Results Using Vertices Coordinates with Noise

bration. Another reason is that it makes the algorithm independent of the font, shape and other features of characters. In theory, both the two methods can rectify signboards taken at a viewing direction of $0^{\circ}$ to $90^{\circ}$ at each direction. At present, we only carried out experiments on images with a viewing direction of less than $45^{\circ}$, because our character extraction experiments show that the detection rate of signboards becomes relatively low when the camera viewing direction is larger than $45^{\circ}$. The third task is to recognize a detected signboard. Here, we employ a simple template matching scheme as shown in Eq.(13) to recognize detected characters. At present, we only limit the detected signboard to matching to a small number of stored images, and the matching strategy is effective. In the future, with the utilization of high efficiency OCR technology, we can apply character recognition not only after distortion rectification, but can also apply it at character candidates stage and stage before rectification. This can help us to dissolve problems due to the ambiguousness of characters and inperfection of rectification, and raise the character detection and recognition rates.

The total image processing time (including detection of characters, distortion rectification and character recognition) differs from image to image. Input images were digitized into 256 grey levels and $640 \times 480$ pixels. Generally it takes about 20-25 seconds for an image using an ONYX workstation. In order to extract character candidates from an input image, we conducted Fourier transformation in each of 1200 subregions (the size of each subregion is $16 \times 16$ pixels). If the FFT process can be replaced by hardware, the time cost will decrease to $10-15$ seconds.

\section{An Example of Application to Indoor} Robot Navigation

We conducted robot navigation experiments in the indoor environment. Here shows an example of robot navigation experiments. The robot is given an environmental map as shown in Fig. 7 (only coordinates, shape and signboard information of each junction are recorded in the map). We use signboards on walls and doors as landmarks to be recognized by the robot automatically. The robot is given such tasks as: 1) go straight and try to find the signboard "図書室"; 2) turn right after finding "図書室"; and 3) go straight until finding the signboard "非常口ヤ" is found. Since the environmental map integrates topological information of the environment, approximate distance information, plus signboard information. When the robot reach a junction of the corridor after moving a proper distance, it will stop and capture an image with its forward-looking camera. By signboard recognition, the robot can confirm its actual position in the environment and adjust its moving direction to the proper one.

As a mobile robot moves through its environment, its actual position and orientation always differs from that it is commanded to hold due to the wheel slippage. In our system, we use walls in the hallway as references to correct the wheel slippage. From the sonar sensor data, we try to adjust the position of the robot and keep it moving in the center of the corridor. Since the camera is fixed on the top of robot, this can also make the camera keep forward-looking. In the real world, compared with the size of a robot, signboards are often placed 


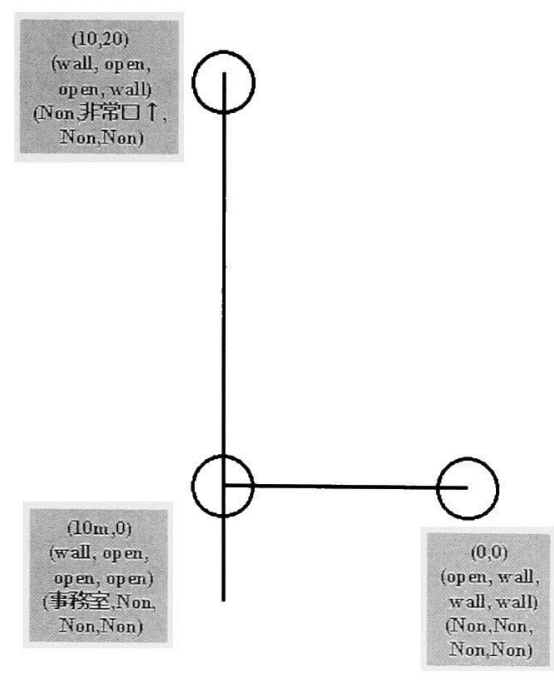

Fig. 7. An Environment Map

at a higher position. This can be resolved by placing the camera at a higher position or using a wide-view camera.

\section{Conclusions}

We presented an approach for finding signboard information in a grey scene image and rectifying distorted signboard images taken from a non-orthogonal viewing direction. By using the features of characters, we extracted character components in a grey scene image. Experimental results showed that the algorithm processes well on both indoor and outdoor real scene images. Based on the projection relationship between the camera coordinate system and the image coordinate system, the viewing direction of a camera relative to a signboard was estimated. Rectification of distorted signboard images made the recognition of characters under a wider range of viewing conditions much easier. In the future, we intent to build a character-base robot navigation system. By using character information as landmarks in the environment, a mobile robot will be able to realize human-like navigation and map generation, which can also benefit human-robot communication.

(Manuscript received July 14, 1999, revised November 12, 1999)

\section{References}

(1) pKeiji Gyohten, Noboru Babaguchi and Tadahiro Kitahashi Region Extraction by Constraint Satisfaction, ACCV'93 Asian Conference on Computer Vision, pp. 846-849 (1993).

(2) Yu Zhong, Kalle Karu, and Anil K. Jain: Locating Text in Complex Color Images, Pattern Recognition, 28(10):15231535, October (1995).

(3) Jun Ohya, Akio Shio, and Shigeru Akamatsu: Recognizing Characters in Scene Images, IEEE Transactions on pattern analysis and machine intelligence, 16(2):214-220, February
(1994)

(4) H. S. Dulimarta and A. K. Jain: Mobile Robot Localization in Indoor Environment, Pattern Recognition, Vol. 30, No. 1 , pp. 99-111 (1997).

(5) J. Miura and Y. Shirai: Vision-Motion Planning for a Mobile Robot under Uncertainty, International Journal of Robot Research, Vol. 16, No. 6, pp. 806-825 (1997)

(6) A. Kosaka and J. Pan: Purdue Experiments in Model-Based Vision for Hallway Navigation, Proc. of Workshop on Vision for Robots in IROS'95 Conference, pp. 87-96 (1995).

(7) Raj Talluri and J. K. Aggarwal: Mobile Robot Self-Location Using Model-Image Feature Correspondence, IEEE Trans. on Robotics and Automation, Vol. 12, No. 1 (1996).

(8) C. Becker, J. Salas, K. Tokusei and J. Latombe: Reliable Navigation Using Landmarks, Proc. of IEEE International Conference on Robotics and Automation, pp. $401-406$ (1995).

(9) Y. Liu, T. Yamamura, N. Ohnishi and N. Sugie: Extraction of Character String Regions from a Scene Image, Trans. IEICE Japan, Vol. J81-D-II, No. 4, pp. 641-650 (1998)(In Japanese).

(10) R .S. Weiss, H. Nakatani, and E. M. Riseman: An Error Analysis for Surface Orientation from Vanishing Points, IEEE trans. on pattern analysis and machine intelligence, Vol. 12, No. 12, pp.1179-1185 (1990).

(1.1) M. Straforini, C. Coelho, M.Campani, and V. Torre: The Recovery and Understanding of a Line Drawing from Indoor Scenes, IEEE trans. on pattern analysis and machine intelligence, Vol. 14, No.2, pp.298-303 (1992).

(12) P. Parodi and G. Piccioli: 3D Shape Reconstruction by Using Vanishing Points, IEEE trans. on pattern analysis and machine intelligence, Vol. 18, No.2, pp.211-217 (1996)

(13) Y. Kawasaki, Y. Yagi, M. Brady and M. Yachida: 3D Road Shape Reconstruction from a Single Image, Proceeding of the 16th Annual Conference of the Robotics Society of Japan Vol.III, pp1577-1578 (1998)(In Japanese).

(14) R.Y. Tsai: Multiframe image point matching and 3-D surface reconstruction, IEEE trans. on Pattern analysis and machine intelligence, Vol.5, No.2, pp.323-345 (1983)

(15) A.Criminisi, I.Reid, A.Zisserman: A Plane Measuring Device, Image and Vision Computer 17, pp. 625-634 (1999)

Yongmei Liu (Non-member) She received the B.E.degree

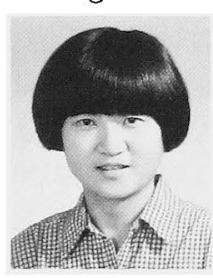
in computer science and engineering from Beijing University of Aeronautics and Astronautics, Beijing, China, in 1990, and M.E. de gree in information engineering from Nagoya University, 1996. She is currently a technical staff at Bio-Mimetic Control Research Center, RIKEN. Her research interests include scene analysis and mobile robot navigation. She is a member of IEEE, IEICE and RSJ.

Tsuyoshi Yamamura (Non-member) He received his BE,

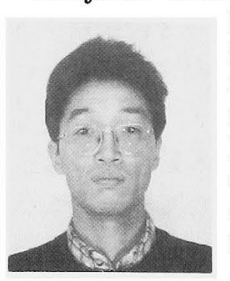
$\mathrm{ME}$ and $\mathrm{Ph} . \mathrm{D}$ degrees from Nagoya University in 1987, 1989 and 1994, respectively. In 1992, he was a Research Associate in the Department of Electronic Information Engineering at Nagoya University. In 1995, he was an Assistant Professor in the Department of Infor mation Engineering, Graduate School of Engineering, Nagoya University. He is presently an Associate Professor in the Faculty of Information Science and Technology, Aichi Prefectural University. $\mathrm{He}$ is engaged in research on Natural Language Processing and Visual Information Processing. $\mathrm{He}$ is a member of IEEE, IEICE and IPSJ. 
Toshimitsu Tanaka (Non-member) He received his BE, $\mathrm{ME}$ and Ph.D Degrees from Nagoya University in 1982, 1984 and 1992, respectively. He was with NTT form 1984 to 1994. From 1994, he has been an associate professor in Nagoya University Computation Center. He is engaged in research on computer graphics. Dr. Tanaka is a member of IPSJ and IEICE.

Noboru Ohnishi (Member) He received the B. Eng., M. Eng. and D. Eng. degrees from Nagoya University, Nagoya, Japan, in 1973, 1975 and 1984, respectively. From 1975 to 1986 he was with the Rehabilitation Engineering Center under the Ministry of Labor. From 1986 to 1989 he was an Assistant Professor in the Department of Electrical Engineering, Nagoya University. From 1989 to 1994, he was an AsUniversity. From 1989 to 1994 , he was an As-
sociate Professor. He is an Professor in the Department of Information Engineering, and concurrently a Head Control Research Center of RIKEN. His research interests include computer-vision and -audition, robotics, bio-cybernetics, and rehabilitation engineering. Dr. Ohnishi is a member of IEEE, IEEJ, IEICE, IPSJ, SICE, JNNS, IIITE and RSJ. 